\title{
Bevacizumab plus chemotherapy as third- or later-line therapy in patients with heavily treated metastatic colorectal cancer
}

This article was published in the following Dove Press journal:

OncoTargets and Therapy

I September 2015

Number of times this article has been viewed

\author{
Qiong Yang ${ }^{1-4, *}$ \\ Chenxi Yin 1,3,4,* \\ Fangxin Liao ${ }^{1,3,4}$ \\ Yuanyuan Huang ${ }^{1,3,4}$ \\ Wenzhuo $\mathrm{He}^{1,3,4}$ \\ Chang Jiang ${ }^{1,3,4}$ \\ Guifang Guo 1,3,4 \\ Bei Zhang ${ }^{1,3,4}$ \\ Liangping $\mathrm{Xia}^{1,3,4}$
}

'VIP Region, Sun Yat-sen University

Cancer Center, Guangzhou,

Guangdong, People's Republic of

China; ${ }^{2}$ Department of Oncology,

Sun Yat-sen Memorial Hospital,

Guangzhou, Guangdong, People's

Republic of China; ${ }^{3}$ State Key

Laboratory of Oncology in South

China, Sun Yat-sen University Cancer

Center, Guangzhou, Guangdong,

People's Republic of China;

${ }^{4}$ Collaborative Innovation Center

for Cancer Medicine, Guangzhou,

Guangdong, People's Republic

of China

*These authors contributed equally to this work
Correspondence: Liangping Xia;

Bei Zhang

VIP Region, Sun Yat-sen University

Cancer Center, Guangzhou, Guangdong

51 0060, People's Republic of China

Tel +86 2087343107

Fax +86 2087343392

Email xialiangping@I63.com;

zhangbei@sysucc.org.cn
Background: Currently available third- or later-line therapy for metastatic colorectal cancer (mCRC) is limited in its efficacy, with a weak survival benefit in patients who progressed after two or more lines of standard therapy. Our retrospective study aimed to explore the value of bevacizumab plus chemotherapy in this setting.

Methods: Patients with mCRC who received fluoropyrimidine, oxaliplatin, and irinotecan as first- and second-line chemotherapy were selected for inclusion. Treatment consisted of bevacizumab plus chemotherapy. Chemotherapy consisted mainly of oxaliplatin, irinotecan, and fluoropyrimidine.

Results: Between February 2010 and December 2012, 35 consecutive patients with mCRC were treated with bevacizumab plus chemotherapy as a third- or later-line treatment. No complete responses, seven partial responses (20\%), 22 stable disease responses (62.9\%), and six progressive disease responses $(17.1 \%)$ were obtained, producing an objective response rate of $20 \%$ and a disease control rate of $82.9 \%$. With a median follow-up of 11.3 months (range: 0.7-48.0 months), the median progression-free survival was 5.98 months (95\% confidence interval: 4.76-7.2 months), and the median overall survival was 14.77 months (95\% confidence interval: $11.45-18.1$ months). In the univariate analysis, patients with a primary colon tumor might have had a longer overall survival than patients with a primary rectal tumor (18.8 months vs 11.1 months, respectively; $P=0.037$ ). Common chemotherapy-related toxicities were nausea/vomiting (48.6\%), fatigue (34.3\%), leucopenia (40\%), neutropenia (42.9\%), and anemia (42.9\%), with one patient with grade 3 neutropenia, and two patients with grade 3 thrombocytopenia. The common bevacizumab-associated toxicity was hypertension $(31.4 \%)$. None of the patients discontinued therapy or died because of bevacizumabassociated toxicities.

Conclusion: Our data showed that adding bevacizumab to third- or later-line therapy might lead to tumor control and improved survival in heavily pretreated mCRC patients. In addition, preliminary data suggested that primary colon cancer was more likely to benefit from bevacizumab-containing regimens. Toxicities were acceptable, and no new toxicity was identified. Further studies are needed to validate these findings.

Keywords: bevacizumab, chemotherapy, metastatic colorectal cancer

\section{Introduction}

Worldwide, colorectal cancer is the third most commonly diagnosed cancer in males and the second most common in females, with an estimated 1.4 million cases and 693,900 deaths occurred in $2012 .{ }^{1}$ Approximately $40 \%-50 \%$ of newly diagnosed patients have metastatic disease, and the prognosis for metastatic colorectal cancer (mCRC) patients remains poor. ${ }^{2}$ At the present, chemotherapy with or without target 
agents was the preferred therapeutic option. Cytotoxic agents for these patients, such as fluoropyrimidines, oxaliplatin, and irinotecan (used in combination and sequentially), prolong progression-free survival (PFS) and overall survival (OS). ${ }^{3}$ Furthermore, monoclonal antibodies targeting vascular endothelial growth factor (VEGF; bevacizumab) and monoclonal antibodies targeting epidermal growth factor receptor (EGFR; cetuximab and panitumumab, specific for $R A S$ wild-type tumors) have increased the median OS to nearly 30 months. ${ }^{4}$ However, many patients will experience disease progression after two or more lines of standard therapy and still have a good performance status. Therefore, it is necessary to explore additional treatments for these patients.

To the best of our knowledge, at least three randomized controlled trials (RCTs) have explored the role of third- or later-line therapy with a survival benefit in mCRC that has progressed after two or more lines of standard therapy. CORRECT first reported that regorafenib might add a survival benefit in these patients, with an objective response rate (ORR) of $1 \%$, a PFS of 1.9 months and an OS of 6.4 months. $^{2}$ In 2015 , at the American Society of Clinical Oncology Gastrointestinal Symposium, Xu et al reported that famitinib also shows a PFS advantage over best supportive care (BSC) in patients with mCRC that progresses after all approved standard therapies (2.8 months vs 1.5 months, respectively; $P=0.0053) .{ }^{5}$ In a Japanese Phase II trial, TAS-102 showed a small superior efficacy with an ORR of $1 \%$, a PFS of 2 months, and an OS of 9 months. ${ }^{6}$ Nevertheless, the absolute survival benefit from both tyrosine kinase inhibitors (TKIs) and TAS-102 is limited. Much effort is needed to explore more effective therapies and improve the survival of these patients.

Bevacizumab is a recombinant, humanized monoclonal antibody targeting VEGF A (VEGF-A), which is a key mediator of tumor angiogenesis. In mCRC, bevacizumab has shown excellent efficacy in both first- and second-line settings in combination with either irinotecan- or oxaliplatinbased chemotherapy. ${ }^{7,8}$ The ML18147 (TML) and BEBYP trials further demonstrated that bevacizumab can be continued after failing a first-line bevacizumab-containing regimen; these trials showed a significant improvement in PFS and OS. ${ }^{9,10}$ However, data on the efficacy of bevacizumab in chemorefractory mCRC patients are still limited, ${ }^{11-17}$ and no data have ever been reported in Chinese patients in this setting.

The current retrospective study was intended to evaluate the efficacy and safety of bevacizumab plus chemotherapy in Chinese patients with $\mathrm{mCRC}$ who have failed two or more lines of standard therapy.

\section{Methods}

\section{Patients}

Histologically confirmed and measurable stage IV CRC patients who were heavily pretreated with oxaliplatincontaining and irinotecan-containing chemotherapy at Sun Yet-sen University Cancer Center between February 2010 and December 2012 were retrospectively reviewed. Prior target drug treatment, such as bevacizumab, cetuximab, and panitumumab, was allowed. Other criteria for eligibility were 1) Eastern Cooperative Oncology Group (ECOG) performance scores (PSs) of 0,1 , or $2 ; 2$ ) adequate hepatic function (bilirubin $<2.0 \mathrm{mg} / \mathrm{dL}$ and transaminases levels $<3$ times the upper normal limit [five times for patients with liver metastasis]); 3) adequate renal function (creatinine $<1.5 \mathrm{mg} / \mathrm{dL}$ ); 4) adequate bone marrow function (absolute neutrophil count $[\mathrm{ANC}]>1,500 / \mu \mathrm{L}$, hemoglobin $>9.0 \mathrm{~g} / \mathrm{dL}$, and platelets $>75,000 / \mu \mathrm{L})$; and 5) a life expectancy of $>3$ months. Written informed consent was required before chemotherapy.

Exclusion criteria included nonhealing wounds, ulcers, bone fractures, thromboembolism that required therapeutic anticoagulation, or major surgery within 6 weeks. Other exclusion criteria were uncontrolled hypertension, bleeding diathesis, active or recent cardiovascular disease or cerebrovascular accident, and pregnancy or lactation in women. The pretreatment characteristics of the patients are presented in Table 1.

\section{Treatment}

The Institutional Review Board of the Sun Yat-sen Cancer Center approved this retrospective study. After careful review, 35 patients met the eligibility criteria and were included in this retrospective study. All the patients received the first dose of bevacizumab after February 2010, when bevacizumab was approved for mCRC in People's Republic of China.

Among the 35 patients, 13 received bevacizumab plus FOLFOX, 17 received bevacizumab plus FOLFIRI, two received bevacizumab plus XELOX, one received bevacizumab plus XELIRI, one received bevacizumab plus irinotecan, and one received bevacizumab plus gemcitabine and raltitrexed. Bevacizumab at a dose of $5 \mathrm{mg} / \mathrm{kg}$ or $7.5 \mathrm{mg} / \mathrm{kg}$ was administered intravenously (IV) over 90 minutes every 2 or 3 weeks prior to the administration of chemotherapy. FOLFIRI consisted of irinotecan $\left(180 \mathrm{mg} / \mathrm{m}^{2}\right)$ IV over 2 hours and leucovorin ( $\left.400 \mathrm{mg} / \mathrm{m}^{2}\right)$ IV over 2 hours, followed by an IV bolus of fluorouracil (5-FU) $\left(400 \mathrm{mg} / \mathrm{m}^{2}\right)$ and 46 hours of continuous 5 -FU IV $\left(2,400 \mathrm{mg} / \mathrm{m}^{2}\right)$. FOLFOX consisted of oxaliplatin $\left(85 \mathrm{mg} / \mathrm{m}^{2}\right)$ IV over 2 hours and leucovorin 
Table I Baseline demographics and clinical characteristics

\begin{tabular}{|c|c|}
\hline Characteristics & No of patients, $\mathbf{N}(\%)$ \\
\hline Total & 35 \\
\hline \multicolumn{2}{|l|}{ Sex } \\
\hline Men & $27(77.1)$ \\
\hline Women & $8(22.9)$ \\
\hline \multicolumn{2}{|l|}{ Age (years) } \\
\hline Median (range) & $56(29-80)$ \\
\hline$\leq 65$ & $30(85.7)$ \\
\hline$>65$ & $5(14.3)$ \\
\hline \multicolumn{2}{|l|}{ ECOG PS } \\
\hline 0 & I (2.9) \\
\hline I & $33(94.3)$ \\
\hline 2 & I (2.9) \\
\hline \multicolumn{2}{|l|}{ Primary tumor site } \\
\hline Colon & $24(68.6)$ \\
\hline Rectum & II (3I.4) \\
\hline \multicolumn{2}{|l|}{ No of metastatic sites } \\
\hline I & $2(5.7)$ \\
\hline 2 & $0(0)$ \\
\hline$\geq 3$ & $33(94.3)$ \\
\hline \multicolumn{2}{|l|}{ KRAS status } \\
\hline Wild-type & $17(48.6)$ \\
\hline Mutation type & $10(28.6)$ \\
\hline Unknown & $8(22.8)$ \\
\hline \multicolumn{2}{|c|}{ Chemotherapy associated with BV } \\
\hline Oxaliplatin-containing & $15(42.9)$ \\
\hline Irinotecan-containing & $19(54.3)$ \\
\hline Other & I (2.8) \\
\hline \multicolumn{2}{|l|}{ Line of bevacizumab } \\
\hline 3 & $29(82.9)$ \\
\hline$\geq 4$ & $6(17.1)$ \\
\hline \multicolumn{2}{|l|}{ Previous target treatment } \\
\hline Anti-VEGF & $9(25.7)$ \\
\hline Anti-EGFR & $10(28.6)$ \\
\hline Neither & $16(45.7)$ \\
\hline \multicolumn{2}{|l|}{ Pretreatment LDH } \\
\hline Normal & $20(57.1)$ \\
\hline Abnormal & $13(37.2)$ \\
\hline Not reported & $2(5.7)$ \\
\hline
\end{tabular}

Abbreviations: BV, bevacizumab; ECOG, Eastern Cooperative Oncology Group; EGFR, epidermal growth factor receptor; KRAS, Kirsten rat sarcoma viral oncogene; LDH, lactate dehydrogenase; PS, performance status; VEGF, vascular endothelial growth factor.

$\left(400 \mathrm{mg} / \mathrm{m}^{2}\right) \mathrm{IV}$ over 2 hours, followed by an IV bolus of 5 -FU $\left(400 \mathrm{mg} / \mathrm{m}^{2}\right)$ and 46 hours of continuous 5-FU IV $\left(2,400 \mathrm{mg} / \mathrm{m}^{2}\right)$. XELOX consisted of oxaliplatin at a dose of $130 \mathrm{mg} / \mathrm{m}^{2}$ as 2-6 hours of infusion on day 1 and Xeloda at a dose of $1 \mathrm{~g} / \mathrm{m}^{2}$ twice daily on day 1 to day 14 every 3 weeks. XELIRI consisted of irinotecan at a dose of $125 \mathrm{mg} / \mathrm{m}^{2}$ as a 90-minute infusion on day 1 and day 8 and Xeloda at a dose of $1 \mathrm{~g} / \mathrm{m}^{2}$ twice daily on day 1 to day 14 every 3 weeks. Threeweekly irinotecan was at a dose of $350 \mathrm{mg} / \mathrm{m}^{2}$. Gemcitabine and raltitrexed consisted of gemcitabine at a dose of $1 \mathrm{~g} / \mathrm{m}^{2}$ as a 30 minute infusion on day 1 and day 8 and raltitrexed at a dose of $3 \mathrm{mg} / \mathrm{m}^{2}$ as a 15 minute infusion on day 1 every 3 weeks. The bevacizumab plus chemotherapy treatment was continued until disease progression or intolerance of therapy.

\section{Assessment}

The primary endpoint was OS, and secondary endpoints were ORR, PFS, and toxicity. Descriptive statistics were reported as proportions and medians. Tumor responses were assessed by RECIST 1.1 criteria every 6-8 weeks. Radiologic evaluation consisted of chest and abdominopelvic computed tomography. PFS was defined as the time from the beginning of bevacizumab as the third- or later-line treatment to disease progression or death. OS was defined as the time from the beginning of bevacizumab as the third- or later-line treatment to death from any cause. Toxicity evaluations were based on the NCI Common Toxicity Criteria for Adverse Events (CTCAE), version 4.0.

\section{Statistical analysis}

Survival curves were estimated using the Kaplan-Meier method. All the analyses were performed using SPSS 16.0 for Windows. A $P$-value less than 0.05 was considered to indicate statistical significance.

\section{Results \\ Patient characteristics}

Between February 2010 and December 2012, 35 consecutive patients with $\mathrm{mCRC}$ were treated with bevacizumab plus chemotherapy (mainly oxaliplatin- or irinotecan-containing regimens) as a third- or later-line treatment. Until April 30, 2015, the median follow-up time was 11.3 months (range: 0.7-48.0 months). The median patient age was 56 years (range: 29-80 years). The ECOG performance status was 0 or 1 in $97.2 \%$ of the patients. Thirty-three (94.3\%) patients had more than three metastatic sites. The previous chemotherapy regimens were irinotecan-containing or oxaliplatincontaining in 30 patients $(85.7 \%)$, such as FOLFOX, FOLFIRI, and XELOX. The dosage, chemotherapy interval, and cycles were strictly based on the guidelines. Prior to this study, 19 patients had received anti-VEGF- or anti-EGFRcontaining therapy (Table 1). All 35 patients identified in this study had progressed on prior therapy according to the RECIST 1.1 evaluation standard. Therefore, these patients were considered as treatment-refractory cases in this study.

\section{Efficacy}

Of the 35 patients, $100 \%(35 / 35)$ and $62.9 \%(22 / 35)$ of patients had experienced progression or died at the last 
Table 2 Response to treatment

\begin{tabular}{ll}
\hline Response & Number (\%) \\
\hline Complete response (CR) & $0(0)$ \\
Partial response (PR) & $7(20)$ \\
Stable disease (SD) & $22(62.9)$ \\
Progressive disease & $6(17.1)$ \\
Not assessable & $0(0)$ \\
Objective response (CR + PR) & $7(20)$ \\
Disease control (CR + PR + SD) & $30(82.9)$ \\
\hline
\end{tabular}

follow-up. The median number of cycles of bevacizumab treatment was 6 (range: 2-17). Treatment discontinuation was caused by disease progression $(29 / 35,82.9 \%)$, inability to tolerate treatment $(2 / 35,5.7 \%)$, palliative surgery $(3 / 35$, $8.6 \%)$, or another reason $(1 / 35,2.9 \%)$. The response rates were as follows: no patient had a complete response, seven patients had a partial response (PR) (20\%), 22 patients had stable disease $(62.9 \%)$, and six patients had progressive disease (17.1\%), giving an ORR of $20 \%$ and a disease control rate of $82.9 \%$ (Table 2). The median PFS was 5.98 months with a $95 \%$ confidence interval of 4.76-7.2 months. The median OS was 14.77 months with a $95 \%$ confidence interval of 11.45-18.1 months (Figure 1). In the univariate analysis, the patient's age, sex, number of metastatic sites, status of Kirsten rat sarcoma viral oncogene (KRAS) mutation, prior anti-VEGF or anti-EGFR treatment, and pretreatment lactate dehydrogenase (LDH) level were not associated with PFS or OS. The OS was 18.56 and 13.77 months for patients treated with or without prior anti-VEGF, respectively; however, there was no statistically significant difference $(P=0.58)$. However, the ORR (all PR) was $42.9 \%(6 / 14)$ in pretreatment high-LDH-level patients, which was higher than the $4.8 \%(1 / 21)$ ORR in pretreatment low-LDH-level patients $(P=0.01)$. Patients with a primary colon tumor may have had a longer OS than patients with a primary rectal tumor, but the PFS was not prolonged (median OS: 18.8 months vs 11.1 months, respectively; $P=0.037$ ).

\section{Safety and toxicity}

The 35 patients received 242 cycles of chemotherapy. There were no treatment-related deaths. Common chemotherapyrelated toxicities were nausea/vomiting $(48.6 \%)$, fatigue (34.3\%), leucopenia (40\%), neutropenia (42.9\%), and anemia (42.9\%). Grade 3 neutropenia developed in one patient (2.9\%), and thrombocytopenia developed in two patients (5.7\%). Grade 3 diarrhea and grade 3 aminotransferase elevation were noted in one patient, respectively. One patient discontinued therapy because of the intolerance of thrombocytopenia, and the other patient discontinued because of diarrhea. Hypertension was the most common bevacizumab-associated toxicity (31.4\%). Grade 1 bleeding, and grade 1 proteinuria occurred in one patient. No grade 3 or 4 bevacizumab-associated toxicities occurred. Bowel perforation and thrombosis/embolism were not observed (Table 3).

\section{Discussion}

Many mCRC patients still have good performance status to tolerate further treatment after standard first-line and
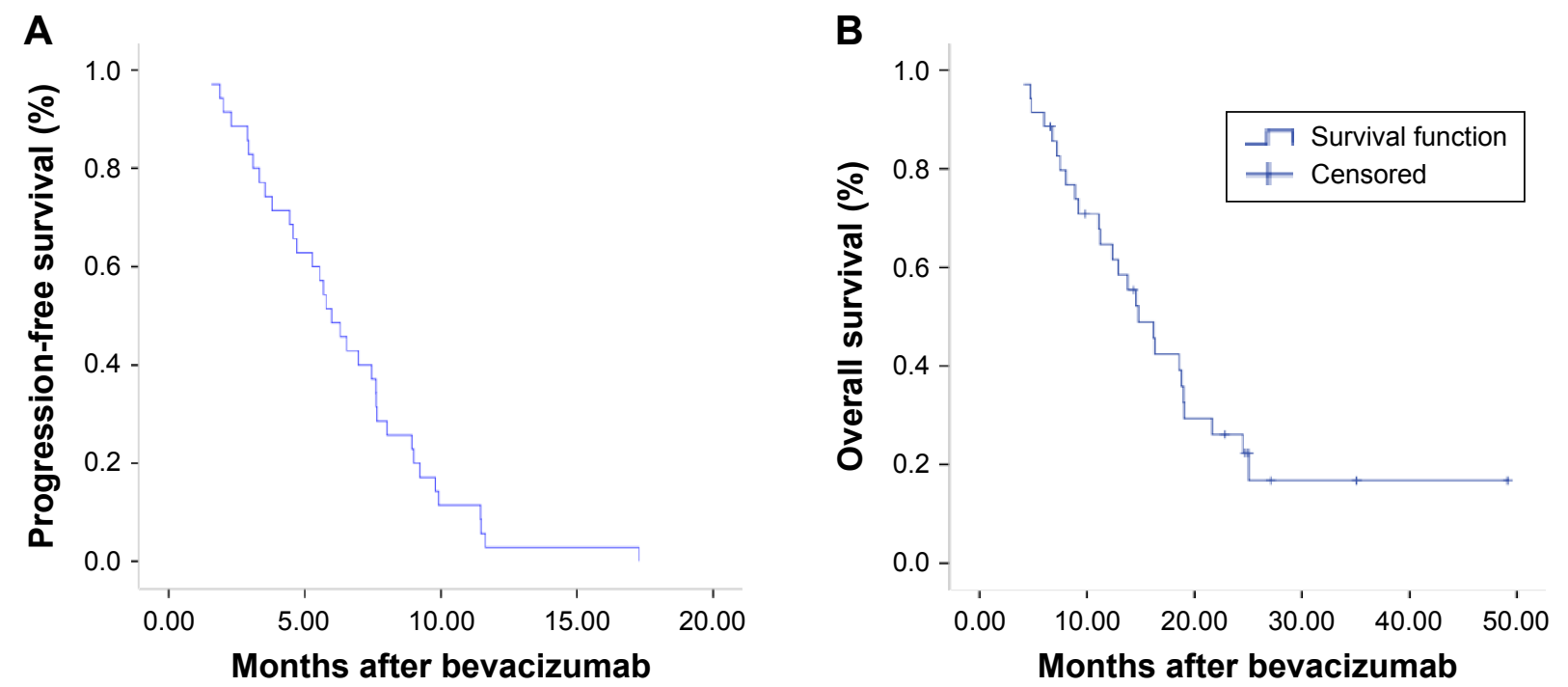

Figure I Kaplan-Meier estimates of progression-free (A) and overall survival (B).

Notes: (A) Median progression-free survival: 5.98 months, 95\% confidence interval: 4.76-7.2 months. (B) Median overall survival: I4.77 months, $95 \%$ confidence interval: II.45-18.1 months. 
Table 3 Toxicity of bevacizumab plus chemotherapy in third- or later-line therapy

\begin{tabular}{lll}
\hline Adverse events & All grades, N (\%) & Grade 3/4, N (\%) \\
\hline $\begin{array}{ll}\text { Hematologic } \\
\text { Leucopenia }\end{array}$ & $14 / 35(40)$ & 0 \\
Neutropenia & $15 / 35(42.9)$ & $1 / 35(2.9)$ \\
Anemia & $15 / 35(42.9)$ & 0 \\
Thrombocytopenia & $4 / 35(11.4)$ & $2 / 35(5.7)$ \\
Nonhematologic & & \\
Fatigue & I2/35 (34.3) & 0 \\
Nausea/vomiting & $17 / 35(48.6)$ & 0 \\
Diarrhea & $7 / 35(20)$ & $1 / 35(2.9)$ \\
Mucositis & $4 / 35(11.4)$ & 0 \\
Liver toxicity & $8 / 35(22.9)$ & $1 / 35(2.9)$ \\
Bevacizumab-associated AE & & \\
Bleeding & $2 / 35(5.7)$ & 0 \\
Gl perforation/fistula & 0 & 0 \\
Thrombosis/embolism & 0 & 0 \\
Hypertension & II/35 (3I.4) & 0 \\
Proteinuria & $2 / 35(5.7)$ & 0 \\
\hline
\end{tabular}

Abbreviations: $\mathrm{AE}$, adverse event; $\mathrm{Gl}$, gastrointestinal.

second-line therapies, but the alternative third- or later-line therapeutic regimens are limited, and the efficacy has been discouraging. Regorafenib was accepted as the third-line therapy based on better survival than BSC, as it showed a discouraging result, with a PFS of 1.9 months and an OS of 6.4 months. ${ }^{2}$ Famitinib and TAS-102 also did not show better efficacy, with a PFS of 2 months and an OS of 6-9 months. ${ }^{5,6}$ Compared with the low ORR (1\%-2.2\%) and small survival benefit in these three RCTs, our study showed a higher response rate with a PR of $20 \%$, a longer median PFS of 5.98 months and a median OS of 14.77 months.

Prior to our study, several small-sample studies including 19-100 patients explored the role of bevacizumab plus chemotherapy as third- or later-line therapy in $\mathrm{mCRC}$ patients who progressed after all standard treatments. The ORR was from $0 \%$ to $28.5 \%$ (median, $9 \%$ ), the PFS was from 3.5 to
8.9 months (median, 3.9 months), and the OS was from 7.7 to 13.8 months (median, 9.5 months). The details are shown in Table $4 .{ }^{11-17}$ Together with those studies, our data suggest that adding bevacizumab to third- or later-line therapy in mCRC patients may lead to tumor control and improved survival. The following reasons may contribute to the potential advantages of bevacizumab in third- or later-line therapy. First, bevacizumab not only inhibits neoangiogenesis, but also normalizes the surviving tumor blood vessels. The later effect results in the accumulation of cytotoxic agents in the tumor, which may enhance the efficacy of chemotherapy. Second, bevacizumab adding a survival advantage as a part of cross-line treatment might imply treatment failure mainly due to resistance to cytotoxic agents but not to anti-angiogenesis drugs. ${ }^{9}, 10$ Moreover, the success of bevacizumab in first- and second-line treatment ${ }^{7,8}$ and the success of regorafenib and famitinib in later-line therapy ${ }^{2,5}$ suggest that anti-angiogenesis may play a role throughout the whole course of treatment. After an indirect comparison of later-line treatments, the promising survival benefit of bevacizumab compared with regorafenib or famitinib suggested not only that head-to-head RCTs comparing the monoclonal antibodies and TKIs are urgently needed, but also that anti-angiogenesis should be combined with chemotherapy, rather than utilized alone, in patients with a good PS in the later-line setting.

In the subgroup analysis, patients with a primary colon tumor may have had a longer OS than those with a primary rectal tumor, which is in accordance with the regorafenib data from the CORRECT trial. ${ }^{2}$ In our study, PFS before the beginning of salvage therapy, pretreatment target drugs, cycles of bevacizumab, chemotherapy regimen, and performance status in salvage therapy were similar in patients with different primary tumor sites. A subgroup analysis from a Phase III trial (FIRE-3), which compared head-to-head

Table 4 Historical data on third- or later-line treatment

\begin{tabular}{|c|c|c|c|c|c|c|c|c|c|}
\hline References & $\begin{array}{l}\text { Publication } \\
\text { year }\end{array}$ & $\begin{array}{l}\text { Type of } \\
\text { study }\end{array}$ & $\mathbf{N}$ & $\begin{array}{l}\text { Treatment } \\
\text { line }\end{array}$ & $\begin{array}{l}\text { Treatment } \\
\text { regimen }\end{array}$ & ORR (\%) & DCR (\%) & TTP/PFS & OS \\
\hline Grothey et $\mathrm{al}^{2}$ & 2013 & Phase III & 255 & Third or later & Best supportive care & 0.4 & 15.4 & 1.7 & 5.0 \\
\hline Grothey et $\mathrm{al}^{2}$ & 2013 & Phase III & 505 & Third or later & Regorafenib & 1.0 & 42.0 & 1.9 & 6.4 \\
\hline Emmanouilides et al" & 2004 & Cohort study & 19 & Third & $\mathrm{BV}+5-\mathrm{FU} / \mathrm{LV}$ & 0 & 47.4 & 3.7 & NA \\
\hline Chen et $\mathrm{al}^{12}$ & 2006 & Phase II & 100 & Third & $\mathrm{BV}+5-\mathrm{FU} / \mathrm{LV}$ & 4.0 & 50 & 3.7 & 9.1 \\
\hline Kwon et $\mathrm{al}^{13}$ & 2007 & Pilot study & 14 & Third & $\mathrm{BV}+\mathrm{FOLFIRI}$ & 28.5 & 85.7 & 3.9 & 10.9 \\
\hline Kang et al ${ }^{14}$ & 2009 & Retrospective & 42 & Third or later & BV+FOLFOX/FOLFIRI & 9.5 & 61.9 & 5.3 & 9.5 \\
\hline Geva et al ${ }^{15}$ & 2013 & Retrospective & 46 & Third or later & BV+chemotherapy & 22 & 83 & 8.9 & 13.8 \\
\hline Vincenzi et $\mathrm{al}^{16}$ & 2009 & Phase II & 48 & Fourth & $\mathrm{BV}+5-\mathrm{FU} / \mathrm{LV}$ & 6.25 & 36.65 & 3.5 & 7.7 \\
\hline Larsen et $\mathrm{al}^{17}$ & 2012 & Retrospective & 34 & Fourth & $\mathrm{BV}+$ Xeloda & 9 & 71 & 5.4 & 12.2 \\
\hline
\end{tabular}

Abbreviations: BV, bevacizumab; 5-FU, Fluorouracil; DCR, disease control rate; NA, not assessed; ORR, objective response rate; OS, overall survival; PFS, progression-free survival; TTP, time to progress; LV, leucovorin; FOLFIRI, Irinotecan plus Fluorouracil plus leucovorin; FOLFOX, Oxaliplatin plus Fluorouracil plus leucovorin. 
bevacizumab with cetuximab in first-line therapy, showed that a primary rectal tumor might benefit from a cetuximabcontaining regimen. ${ }^{4}$ In summary, these studies suggested that a benefit from the target drugs was associated with the primary tumor site. Presently, the reason that primary colon cancer benefits from anti-VEGFR pathway therapy more than primary rectal cancer has not been elucidated. It was reported that VEGF-A, the target of bevacizumab, is present in higher levels in left-sided colon and rectal cancers than in right-sided colon cancers. ${ }^{18,19}$

It has been reported that pretreatment LDH levels can predict the efficacy of first-line bevacizumab-based therapy in mCRC patients. ${ }^{20,21}$ Our data did not show that patients with a pretreatment high LDH level have a longer OS when treated with third- or later-line bevacizumab-containing therapy. However, the response rate was $42.9 \%$ (6/14) in pretreatment high-LDH-level patients, which was higher than the $4.8 \%$ $(1 / 21)$ response rate in pretreatment low-LDH-level patients. Theoretically, LDH can increase the accumulation of lactate in tumors and create an acidic tumor microenvironment, which enhances pericyte deficiency, neovascularization, and sensitivity to antiangiogenesis agents. ${ }^{22,23}$ Our study could not predict a role for $\mathrm{LDH}$ in third- or later-line therapy. This might be explained by the limitation of the small sample and retrospective nature of our study. A larger sample and a prospective study are needed to further answer this question.

The toxicity profile of bevacizumab plus chemotherapy seemed to be similar to those observed in previous studies, ${ }^{11-17}$ which included chemotherapy-related and bevacizumab-related toxicities. The common adverse events related to chemotherapy were leucopenia, anemia, fatigue, and nausea/vomiting. The common adverse events related to bevacizumab were hypertension, bleeding, and proteinuria. These toxicities were tolerable, and no dosage was adjusted or therapy was interrupted by the toxicity. Two patients discontinued therapy because of the intolerance of thrombocytopenia and diarrhea. No gastrointestinal perforations and embolisms occurred, most likely due to the small number of patients. No patients discontinued therapy because of bevacizumab-associated toxicities.

Several limitations hampered the generalization of the present results to all patients with heavily treated mCRC; these limitations can be partly attributable to the study's retrospective nature. First, selection bias cannot be excluded because only medically fit patients with very a good performance status might have been treated with bevacizumab-containing third- or later-line therapy. This is suggested by the inclusion of only one patient with an
ECOG performance status of 2 in the whole cohort of bevacizumab-treated patients, which might have led to a more favorable OS estimate in these patients. Second, the small cohort in this study may also affect the objectivity of our data. Third, the PFS evaluation could have been biased by the fact that neither independent monitoring nor a centralized review of the radiological responses was performed in our study. Fourth, this study had no data on chemotherapy alone. Nevertheless, our data were equal to or a little better than previous similar data, which were superior to the results of chemotherapy alone in the third- or later-line therapy. ${ }^{2,5}$ Finally, there were insufficient data on toxicity, particularly on peripheral neuropathy associated with oxaliplatin, not necessarily indicating the absence of peripheral neuropathy associated with oxaliplatin, but rather the lack of detailed medical records.

\section{Conclusion}

To the best of our knowledge, this was the first study to explore the role of bevacizumab-containing regimens as third- or laterline therapy in Chinese patients with heavily treated mCRC. Our data showed that adding bevacizumab to third- or later-line therapy may lead to tumor control and improved survival in mCRC patients. In addition, preliminary data suggested that primary colon cancer was more likely to benefit from bevacizumab-containing regimens as third- or later-line therapy. Further studies are needed to validate these findings.

\section{Acknowledgments}

This study was supported by grants from Science and Technology Planning Project of Guangdong Province, People's Republic of China (2011B061300069) and National Natural Science Foundation of China (81272641, 81071872 and 81572409).

\section{Disclosure}

The authors report no conflicts of interest in this work.

\section{References}

1. Torre LA, Bray F, Siegel RL, Ferlay J, Lortet-Tieulent J, Jemal A. Global cancer statistics, 2012. CA Cancer J Clin. 2015;65(2):87-108.

2. Grothey A, Van Cutsem E, Sobrero A, et al. Regorafenib monotherapy for previously treated metastatic colorectal cancer (CORRECT): an international, multicentre, randomised, placebo-controlled, phase 3 trial. Lancet. 2013;381(9863):303-312.

3. Grothey A, Hedrick EE, Mass RD, et al. Response-independent survival benefit in metastatic colorectal cancer: a comparative analysis of N9741 and AVF2107. J Clin Oncol. 2008;26(2):183-189.

4. Heinemann V, von Weikersthal LF, Decker T, et al. FOLFIRI plus cetuximab versus FOLFIRI plus bevacizumab as first-line treatment for patients with metastatic colorectal cancer (FIRE-3): a randomised, open-label, phase 3 trial. Lancet Oncol. 2014;15(10):1065-1075. 
5. Xu RH, Shen L, Wang KM, et al. A randomized, double-blind, parallel-group, placebo-controlled, multicenter, phase II clinical study of famitinib in the treatment of advanced metastatic colorectal cancer. J Clin Oncol. 2015;33(Suppl 3):abstr 513.

6. Yoshino T, Mizunuma N, Yamazaki K, et al. TAS-102 monotherapy for pretreated metastatic colorectal cancer: a double-blind, randomised, placebo-controlled phase 2 trial. Lancet Oncol. 2012;13(10): 993-1001.

7. Venook AP, Niedzwiecki D, Lenz HJ, et al. CALGB/SWOG 80405: Phase III trial of irinotecan/5-FU/leucovorin (FOLFIRI) or oxaliplatin/5-FU/leucovorin (mFOLFOX6) with bevacizumab (BV) or cetuximab (CET) for patients (pts) with KRAS wild-type (wt) untreated metastatic adenocarcinoma of the colon or rectum (MCRC). $J$ Clin Oncol. 2014;32(5S): abstr LBA3.

8. Giantonio BJ, Catalano PJ, Meropol NJ, et al. Bevacizumab in combination with oxaliplatin, fluorouracil, and leucovorin (FOLFOX4) for previously treated metastatic colorectal cancer: results from the Eastern Cooperative Oncology Group Study E3200. J Clin Oncol. 2007; 25(12):1539-1544.

9. Bennouna J, Sastre J, Arnold D, et al. Continuation of bevacizumab after first progression in metastatic colorectal cancer (ML18147): a randomised phase 3 trial. Lancet Oncol. 2013;14(1):29-37.

10. Masi G, Salvatore L, Boni L, et al. Continuation or reintroduction of bevacizumab beyond progression to first-line therapy in metastatic colorectal cancer: final results of the randomized BEBYP trial. Ann Oncol. 2015;26(4):724-730.

11. Emmanouilides C, Pegram M, Robinson R, Hecht R, Kabbinavar F, Isacoff W. Anti-VEGF antibody bevacizumab (Avastin) with 5FU/LV as third line treatment for colorectal cancer. Tech Coloproctol. 2004; 8(Suppl 1):S50-S52.

12. Chen HX, Mooney M, Boron M, et al. Phase II multicenter trial of bevacizumab plus fluorouracil and leucovorin in patients with advanced refractory colorectal cancer: an NCI Treatment Referral Center Trial TRC-0301. J Clin Oncol. 2006;24(21):3354-3360.

13. Kwon HC, Oh SY, Lee S, Kim SH, Kim HJ. Bevacizumab plus infusional 5-fluorouracil, leucovorin and irinotecan for advanced colorectal cancer that progressed after oxaliplatin and irinotecan chemotherapy: a pilot study. World J Gastroenterol. 2007;13(46):6231-6235.
14. Kang BW, Kim TW, Lee JL, et al. Bevacizumab plus FOLFIRI or FOLFOX as third-line or later treatment in patients with metastatic colorectal cancer after failure of 5-fluorouracil, irinotecan, and oxaliplatin: a retrospective analysis. Med Oncol. 2009;26(1):32-37.

15. Geva R, Vecchione L, Tejpar S, Piessevaux H, Van Cutsem E, Prenen H. Bevacizumab plus chemotherapy as salvage treatment in chemorefractory patients with metastatic colorectal cancer. Onco Targets Ther. 2013; 6:53-58.

16. Vincenzi B, Santini D, Russo A, et al. Bevacizumab in association with de Gramont 5-fluorouracil/folinic acid in patients with oxaliplatin-, irinotecan-, and cetuximab-refractory colorectal cancer: a single-center phase 2 trial. Cancer. 2009;115(20):4849-4856.

17. Larsen FO, Boisen MK, Fromm AL, Jensen BV. Capecitabine and bevacizumab in heavily pre-treated patients with advanced colorectal cancer. Acta Oncol. 2012;51(2):231-233.

18. Bendardaf R, Buhmeida A, Hilska M, et al. VEGF-1 expression in colorectal cancer is associated with disease localization, stage, and long-term disease-specific survival. Anticancer Res. 2008;28(6B):3865-3870.

19. Boisen MK, Johansen JS, Dehlendorff C, et al. Primary tumor location and bevacizumab effectiveness in patients with metastatic colorectal cancer. Ann Oncol. 2013;24(10):2554-2559.

20. Scartozzi M, Giampieri R, Maccaroni E, et al. Pre-treatment lactate dehydrogenase levels as predictor of efficacy of first-line bevacizumabbased therapy in metastatic colorectal cancer patients. Br J Cancer. 2012 106(5):799-804.

21. Yin CX, Jiang C, Liao FX, et al. Initial LDH level can predict the survival benefit from bevacizumab in the first-line setting in Chinese patients with metastatic colorectal cancer. Onco Targets Ther. 2014;7: 1415-1422.

22. Lee DC, Sohn HA, Park ZY, et al. A lactate-induced response to hypoxia. Cell. 2015;161(3):595-609.

23. Azuma M, Shi M, Danenberg KD, et al. Serum lactate dehydrogenase levels and glycolysis significantly correlate with tumor VEGFA and VEGFR expression in metastatic CRC patients. Pharmacogenomics. 2007 8(12):1705-1713
OncoTargets and Therapy

\section{Publish your work in this journal}

OncoTargets and Therapy is an international, peer-reviewed, open access journal focusing on the pathological basis of all cancers, potential targets for therapy and treatment protocols employed to improve the management of cancer patients. The journal also focuses on the impact of management programs and new therapeutic agents and protocols on

\section{Dovepress}

patient perspectives such as quality of life, adherence and satisfaction. The manuscript management system is completely online and includes a very quick and fair peer-review system, which is all easy to use. Visit http://www.dovepress.com/testimonials.php to read real quotes from published authors. 